\title{
Energy Efficient Load Balanced Routing in wireless Sensor Networks
}

\author{
S.Saranya ${ }^{1}$, A.Vanathi ${ }^{2}$, M.Ramya ${ }^{3}$, P.Bharanee ${ }^{4}$ \\ Assistant professor, Dept. of ECE, Sri Ramakrishna Engineering College, Coimbatore, Tamilnadu, India ${ }^{1,3,4}$ \\ Assistant professor, Dept. of ECE, Kongu Engineering College, Perundurai, Tamilnadu, India ${ }^{2}$
}

\begin{abstract}
Wireless Sensor Networks plays a vital role in monitoring the real time environment using their selforganized nature. The Wireless Sensor Networks are operated by battery power which constrains the overall lifespan of the network. Different clustering methodologies based on hierarchical routing have been a remedy for these power constrained self-organized networks. Many works for balancing the load in terms of energy are contemplated but the load balancing in the Sensor Networks in terms of traffic still remains as a great challenge. In case of densely deployed networks when the traffic level is very high the real time monitored data gets lost due to limited buffer size in the Wireless Sensor Networks. These problems can be fixed by formulating a new routing protocol called Distributed Load Balanced Routing which can overcome the constrains overall network lifespan and the congestion in the Buffer.
\end{abstract}

Keywords: Wireless Sensor Networks; Gateways; Cluster Heads; Clusters

\section{Introduction}

A Wireless Sensor Network (WSN) is made up of self-organized independent sensors powered by batteries. These sensors are capable of monitoring the real time environmental parameters like temperature, pressure and sound. The applications of the wireless sensors networks are listed as air pollution monitoring, landslide detection, forest fire detection, water quality monitoring etc.,

The energy is the scarcest resource of WSN nodes and it determines the lifetime of WSNs. The WSNs are scattered in various types of environments, which may be hostile or remote regions. The areas of interest where the research is to be performed in the WSNs are

1. Lifetime maximization

2. Robustness and fault tolerance

3. Self-configuration

The lifetime of a network is the minimum lifetime of all of the sensor nodes in the network. The sensor nodes located close to the sink are heavily used to relay data from all network nodes hence; their energy is consumed faster, leading to a non-uniform depletion of energy in the WSN, resulting in decreased lifetime of the network. Network periphery nodes run the risk of rapid energy exhaustion resulting in loss of network connectivity from particular area. This paper aims in formulating a new load balancing routing algorithm which can increase the overall lifetime of the sensor nodes and also ensures the data transfer without packet drop. The load balancing can be justified by using two

scenarios like uniform distribution and random distribution. These scenarios can hold good when the gateway experiences less traffic but in applications like forest fire monitoring when a huge sensors transmit data at a time it faces the problem of packet drop, which can be rectified by using the Distributed Load Balanced Routing (D-LBR) in terms of energy as well as traffic.

The remainder of this paper is organized as follows. Section 2. we put forward the system Architecture of WSN scheme. In Section 3, reviews several cluster based algorithms proposed previously. In Section 4, we investigate our new balanced clustering algorithm.. Finally, conclusions are given in the last section of the paper.

\section{System Architecture}

The general system architecture of the WSNs can be described in the Fig.1 which categorizes the nodes under three groups namely motes or sensor nodes, cluster heads and gateway or sink nodes. 


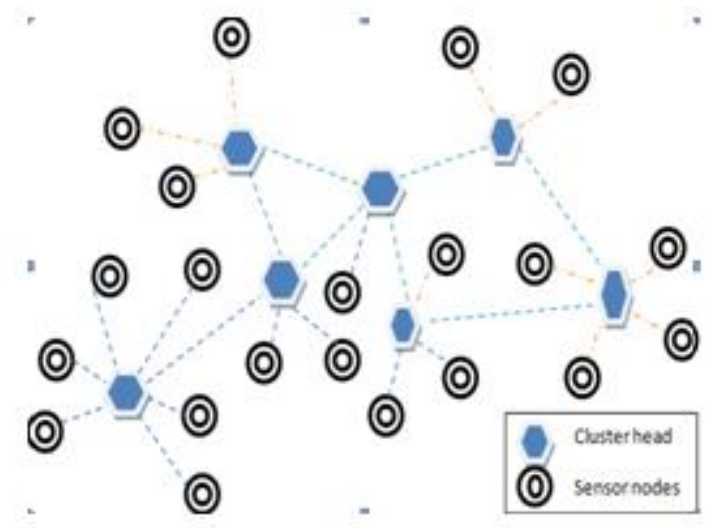

Fig.1 Wireless Sensor Networks self-organization

\section{A. Clusters}

The individual sensors are grouped into several units known as clusters. The cluster formation can change with respect to time depending on the alive sensor nodes at that time. Clusters formation can save energy to a maximum extent instead of individual nodes participating in the data transfer only the Cluster Heads participate from the particular cluster.

\section{B. Cluster Head $(\mathbf{C H})$}

Cluster usually has a controller node, called the cluster head that has a distinguished role. For instance, the cluster head may be responsible for controlling the operation of the sensor nodes in the cluster by setting their configuration parameters, and for aggregating the sensor readings collected from the cluster and storing the result or sending it to the sink or some higher level cluster head.

\section{Gateway or Sink}

A node on the network that serves as a entrance to another network. In WSNs when a sensor is connected to computer it acts as a gateway, which routes the traffic from the sensor network to the server. In other words Sink nodes are the sensors with less energy constrain. Each gateway features a $2.4 \mathrm{GHz}$, IEEE 802.15.4 radio to communicate with up to eight end nodes (in a star topology) or up to 36 WSN measurement nodes (in a mesh topology).

\section{Related Works}

Heinzelman et al proposed LEACH [1] (Low Energy Adaptive Clustering Hierarchy) protocol which comprises of two phases setup and steady state phase. The duration of the steady state phase should be greater than setup phase to enhance the energy efficiency. It depends upon the residual energy for selecting the cluster head selection. In this a cluster head is selected by fixing a threshold and then the clusters are formed by the advertising phase, cluster setup followed by schedule creation. This protocol can hold good for periodical transmission of information.

LEACH protocol suffers due to the overhead caused by the cluster head formation Lindsey et al proposed a protocol named PEGASIS [2,3] (Power Efficient gathering in Sensor Information Systems).It works based on the Greedy Algorithm and chain formation. The chain leader controls the communication order by passing a token among the nodes. The energy efficiency is improved to 100-300\% but it causes time delay due to sequential data transmission and requires data aggregation which leads to inaccuracies.

Manjeshwar et al proposed TEEN [4] protocol (Threshold Sensitive Energy Efficient Sensor Networks) which works on event based delivery in the network. The drawback of TEEN is since its based on fixed threshold it cannot be used for periodical reports.

Manjeshwar et al proposed ATEEN [5] protocol (Adaptive Threshold Sensitive Energy Efficient Sensor Networks) to overcome the defect of TEEN. This protocol can hold good for both periodic and event based information exchange.

In [8], the authors focused on the energy consumption issue in SPEED protocol. Therefore, they proposed to consider the residual energy during the routing decision. It is based on a weight function, which is a combination of three factors: delay, energy and speed. Then, the node with the greatest cost is selected as the next forwarding hop. 
In [9] extending network lifetime issue was also addressed. The contribution of this work is about using data aggregation by one node in each region. The geographical organization and management of each region is done via the Geographical Adaptive Fidelity (GAF) protocol presented in [10].

\section{Load Balanced Routing}

A novel clustering algorithm called DSBCA [6] (Balanced Clustering Algorithm with Distributed SelfOrganization for Wireless Sensor Networks) based on the density of the sensor nodes and distance from their base station a balanced clusters are formed. In the case of uniform distribution the clustering radius in the same layer is identical and the radius of the cluster increases as their distance from the base station increases.

In the case of non-uniform distribution the cluster radius depends on two parameters such as distance from the base station and the connectivity density of the nodes [7].

The more balanced clusters both in energy and position can be created by using the weight factor formulation. The weight factor comprises of residual energy to initial energy of the node, density of the node and number of times the respective node has been selected as cluster head. In this way the overall energy consumption is more balance in the Wireless Sensor Network. The Equation (4.1) formulating the weight factor is each node is given by

$$
\begin{aligned}
& W(u)=\phi \times P\left[D_{k}(u)\right]+\varphi \times P\left[\frac{R_{e}(u)}{E(u)}\right]-\gamma \times P[H(u)] \\
& 0 \leq \phi, \varphi, \gamma \leq 1, \quad \gamma<\phi+\varphi<1
\end{aligned}
$$

Where $\varphi, \square, \gamma$ as the effect factors are defined by specific application, $R_{s}(u)$ is the residual energy of node $\mathrm{u}, \mathrm{E}(\mathrm{u})$ is the initial energy of node $\mathrm{u}, \mathrm{H}(\mathrm{u})$ is the times of the node $\mathrm{u}$ being elected as cluster head. In this way we decrease the prospects of $u$ being elected as cluster head to balance the overall energy consumption.

The Fig. 2 shows the performance comparison of the standard routing protocols like AODV and LEACH with the balanced clustering algorithm. To facilitate the comparison survival time is represented by the number of rounds where each round is evoked by setup phase followed by steady state phase.

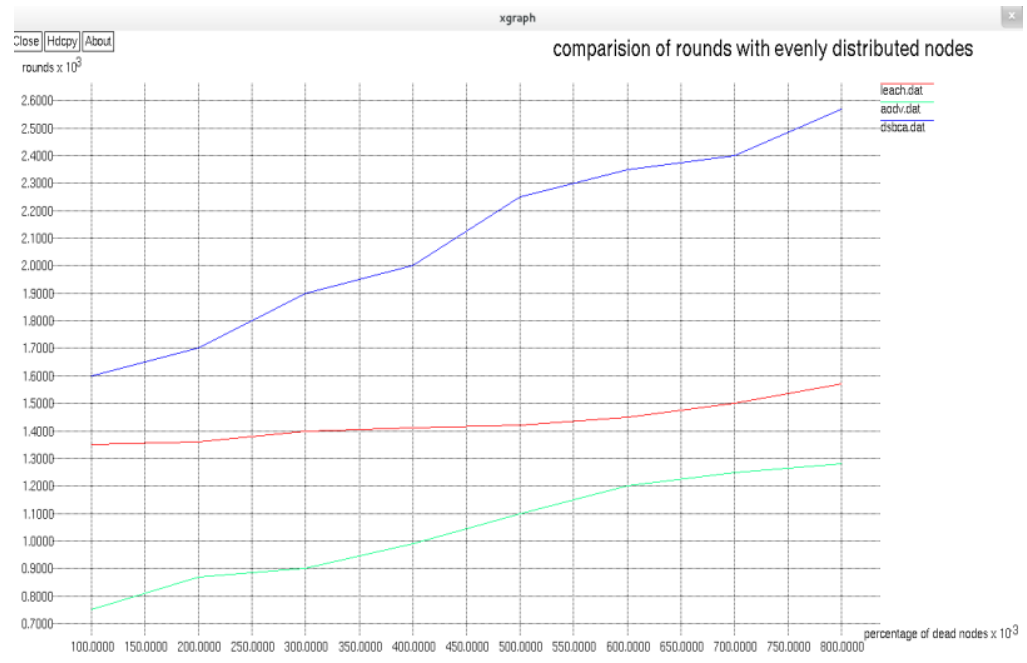

Fig.2 Percentage of dead nodes for evenly distributed Scenario

The Fig. 3 shows the performance comparison in randomly distributed scenario. In both scenarios the overall network lifecycle ends only when $80 \%$ of nodes are dead. When correlating the algorithm of same rounds, the algorithm with low ratio of dead nodes is regarded better. 


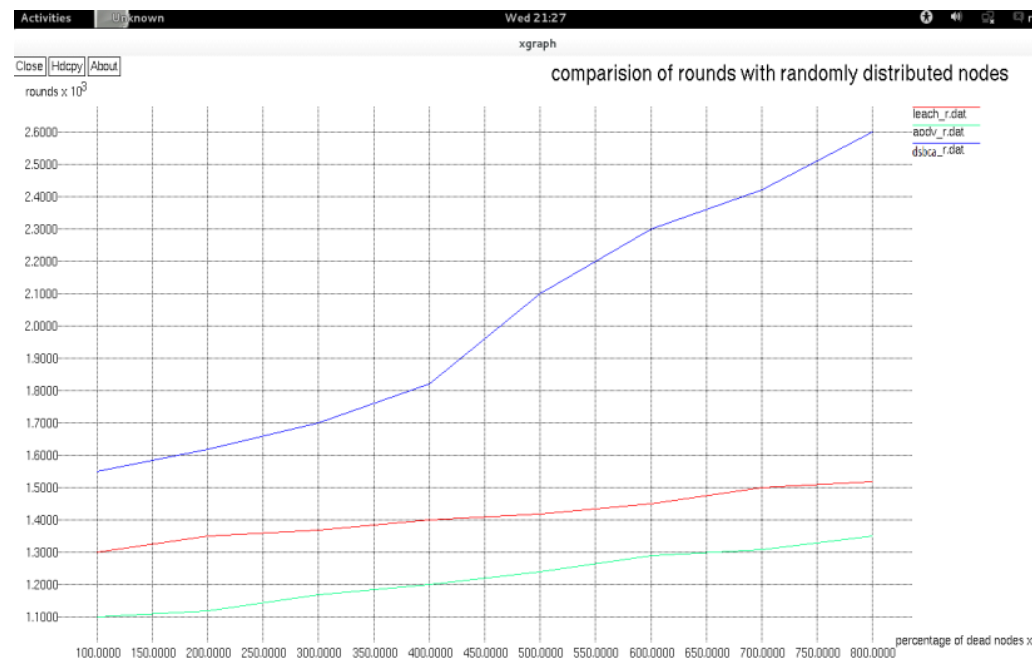

Fig.3 Percentage of dead nodes for randomly distributed Scenario

The Table I, II and III shows $0.1 \%, 0.4 \%$ and $0.8 \%$ of dead nodes for various rounds in both scenarios. The load balancing algorithm holds good for both scenarios resulting in less percentage of dead nodes compared to other routing protocols. This algorithm holds well in terms of energy balancing than other algorithm even when the number of nodes increases but fails to address the problem of balancing the traffic of the nodes. This issue results in the Packet drop as shown in Fig.4.

Table I Percentage Of Dead Nodes: $0.1 \%$

\begin{tabular}{|l|l|l|}
\hline \multirow{2}{*}{ Routing protocol } & No. of Rounds \\
\cline { 2 - 3 } & Even scenario & Random scenario \\
\hline AODV & 750 & 1100 \\
\hline LEACH & 1350 & 1300 \\
\hline DSBCA & 1600 & 1550 \\
\hline
\end{tabular}

Table II Percentage Of Dead Nodes: $0.4 \%$

\begin{tabular}{|l|l|l|}
\hline \multirow{2}{*}{ Routing protocol } & No. of Rounds \\
\cline { 2 - 3 } & Even scenario & Random scenario \\
\hline AODV & 1000 & 1200 \\
\hline LEACH & 1410 & 1400 \\
\hline DSBCA & 2000 & 1820 \\
\hline
\end{tabular}

Table III Percentage Of Dead Nodes: $0.8 \%$

\begin{tabular}{|l|l|l|}
\hline \multirow{2}{*}{ Routing protocol } & No. of Rounds \\
\cline { 2 - 3 } & Even scenario & Random scenario \\
\hline AODV & 1280 & 1350 \\
\hline LEACH & 1580 & 1520 \\
\hline DSBCA & 2550 & 2600 \\
\hline
\end{tabular}

The throughput of DSBCA algorithm holds good when less number of nodes are employed. But when the number of nodes increases from 50,100, 150, 200,250, 300 the packet drop also increases drastically.

Figure 4 shows the comparison of packet drop with number of nodes. When the number of nodes involving in transmission increases resulting in more traffic the real time monitored data gets lost due to the unbalanced load of sensor nodes. 


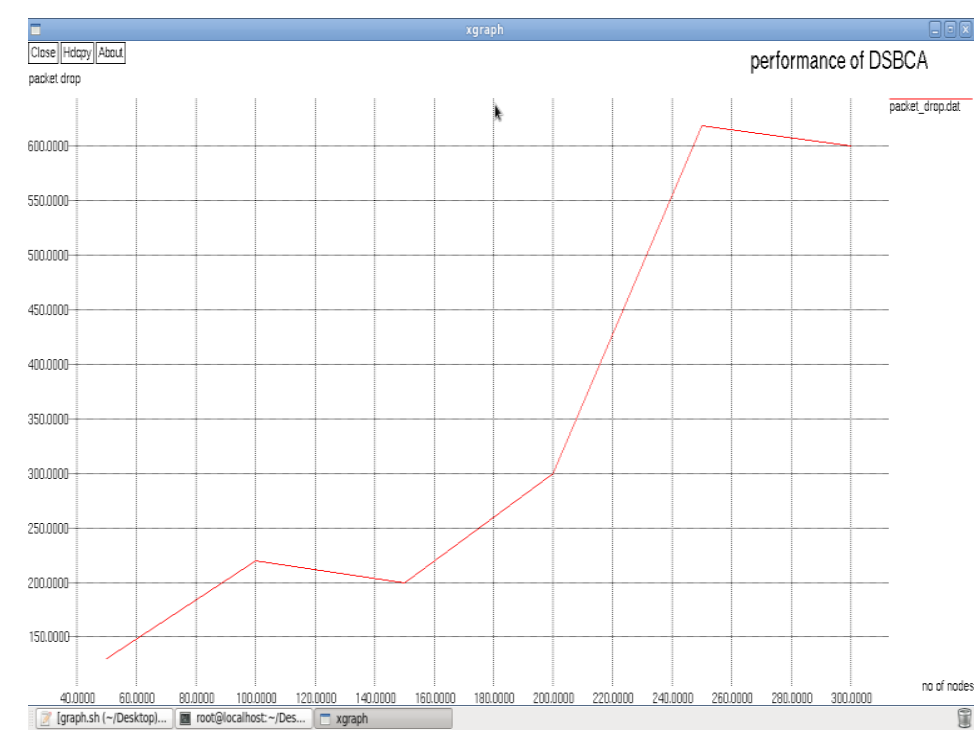

Fig.4 Comparison of Packet drop with number of nodes

\section{A. Reason for load balancing in terms of traffic}

\section{Load Balanced Routing}

The load balancing in terms of traffic is essential mostly at the gateways and cluster heads because they perform the function of aggregating the data from various cluster heads. The sensors cannot have a long buffer due to their limited internal memory. The buffer size of gateways varies according to the electronic gadgets they are connected with sensors.

\section{B. LBR Algorithm}

The Load Balanced Algorithm as shown in fig.5.is invoked when the cluster head and gateway nodes receives data packets from their neighbors. The monitored data in the sensors are routed to the server based on the remnant energy and the buffer size (queue size) of the nodes. Each individual sensor has 16 channels in which only 3 channel are actually used for communication. In the algorithm LIC denotes the Licensing Interference Queue and LQ is the Licensing Queue and $\mathrm{N}_{\mathrm{ch}}^{\mathrm{i}}$ is the current channel of node $\mathrm{N}$.

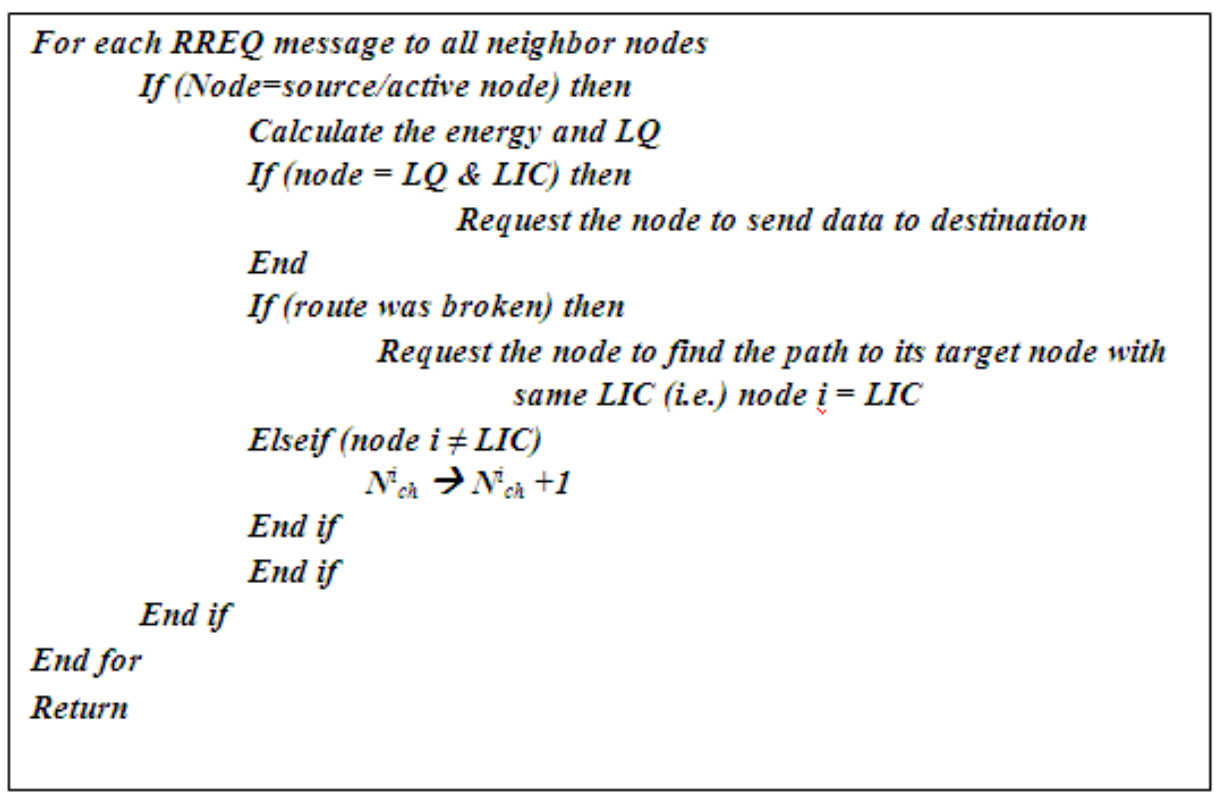

Fig.5 Load Balanced routing Algorithm

The load balancing can be of two types depending on the environment of the gateway configuration. In case of homogenous nature the load sharing is done equally irrespective of configuration whereas in case of heterogeneous environment the load sharing takes places proportionately depending upon the gateway configuration. 
The Fig.6 shows the analysis of the packet drop taking place in the proposed algorithm

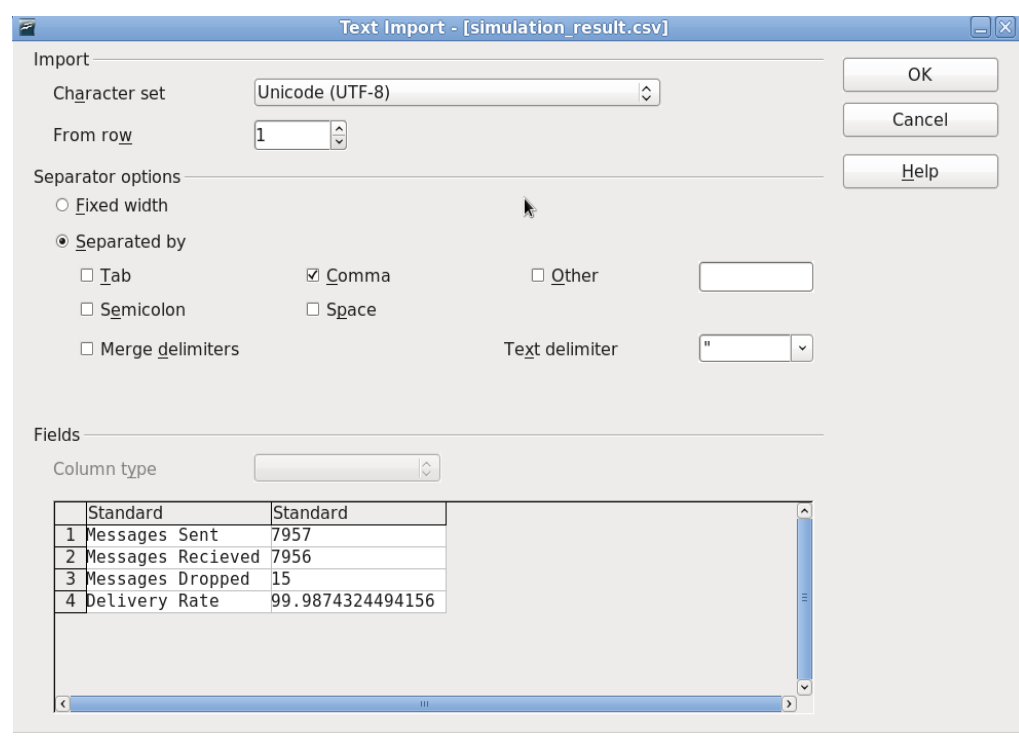

Fig.6 Analysis of packet drop in LBR

The Simulation parameters used for the above mentioned scenarios are given in the Table IV.

Table IV Simulation Parameters

\begin{tabular}{|l|l|}
\hline Number of nodes & $50,100,150 \ldots, 300$ \\
\hline Number of cluster heads & Not constant \\
\hline Number of gateway & 3 \\
\hline Simulation area & $50 \times 50$ \\
\hline Scenarios & $\begin{array}{l}\text { Evenly distributed } \\
\text { Randomly distributed }\end{array}$ \\
\hline Initial energy of sensor nodes & 150 joules \\
\hline Data packet size & 30 bytes \\
\hline Simulation time & 100 sec \\
\hline Movement & Random \\
\hline Transmit Power & $24.75 \mathrm{~mW}$ \\
\hline Receive Power & $13.5 \mathrm{~mW}$ \\
\hline Idle Power & $13.5 \mathrm{~mW}$ \\
\hline Sleep Power & $15 \mu \mathrm{W}$ \\
\hline Routing protocols & AODV \\
& LEACH \\
& DSBCA \\
\hline
\end{tabular}

The delivery rate of the LBR protocol is 99.98 , resulting in a more balanced routing algorithm for sensor networks.

\section{Conclusion}

The energy efficient load balancing algorithm can be formulated by combining the weight factor update of distributed load balanced clustering with the gateway traffic balancing algorithm. This results in extended lifespan of the sensor network till $80 \%$ of nodes are dead and the packet loss with highly deployed environment can be balanced resulting in improved packet delivery ratio up to $90 \%$.

\section{References}

[1]. W. R. Heinzelman, A. Chandrakasan, and H. Balakrishnan. Energy-efficient communication protocol for wireless microsensor networks. In Proceedings of the IEEE Hawaii International Conference on System Sciences, pp. 1-10, Maui, HI, USA, January 2000 .

[2]. S. Lindsey and C. S. Raghavendra. PEGASIS: power efficient gathering in sensor information systems. In Proceedings of the IEEE Aerospace Conference, Big Sky, MT, USA, March 2002.

[3]. S. Lindsey, C. S. Raghavendra, and K. Sivalingam. Data gathering in sensor networks using the energy*delay metric. In Proceedings of the IPDPS Workshop on Issues in Wireless Networks and Mobile Computing, San Francisco, USA, April 2001.

[4]. A. Manjeshwar and D. P. Agrawal. TEEN: a protocol for enhanced efficiency in wireless sensor networks. In Proceedings of the 1st International Workshop on Parallel and Distributed Computing Issues in Wireless Networks and Mobile Computing, San Francisco, USA, April 2001. 
[5]. A. Manjeshwar and D. P. Agrawal. APTEEN: a hybrid protocol for efficient routing and comprehensive information retrieval in wireless sensor networks. In Proceedings of the 2nd International Workshop on Parallel and Distributed Computing Issues in Wireless Networks and Mobile Computing, Ft. Lauderdale, FL, USA, April 2002.

[6]. Ying Liao, Huan Qi, and Weiqun Li Load-Balanced Clustering Algorithm With Distributed Self-Organization for Wireless Sensor Networks IEEE Sensors Journal, vol. 13, no. 5, may 2013

[7]. Bagci H. and Yazici A (2010), 'An energy aware fuzzy unequal clustering algorithm for wireless sensor networks,' Proc. IEEE Int. Conf. Fuzzy Syst., pp. 1-8.

[8]. Kordafshari, M.S., Pourkabirian, A., Faez, K. and Rahimabadi, A.M. (2009), "Energy-Efficient SPEED Routing Protocol for Wireless Sensor Networks", Proceedings of the Fifth Advanced International Conference on Telecommunications, Washington, DC, USA,2009, pp. 267-271.

[9]. Roustaei, R., Fakhr, F.Y. and Movaghar, A. (2010), "The Addition of Data Aggregation to SPEED Routing Algorithm While Keeping the Functionality of Available Techniques", Second International Conference on Future Networks, Malayer, Iran, pp. 349353.

[10]. Xu, Y., Heidemann, J. and Estrin, D. (2001), "Geography-informed energy conservation for ad hoc routing", Proceedings of the 7th annual international conference on Mobile computing and networking, ACM New York, NY, USA, pp. 70-84. 